\title{
Optimization of superconducting properties of the stoichiometric $\mathrm{CaKFe}_{4} \mathrm{As}_{4}$
}

\author{
Shiv J. Singh,,${ }^{1, *}$ Simon J. Cassidy, ${ }^{2}$ Matthew Bristow, ${ }^{1}$ Stephen J. Blundell, ${ }^{1}$ Simon J. Clarke, ${ }^{3}$ and Amalia I. Coldea ${ }^{1} \dagger$ \\ ${ }^{1}$ Clarendon Laboratory, Department of Physics, University of Oxford, Parks Road, Oxford OX1 3PU, UK \\ ${ }^{2}$ Department of Chemistry, University of Oxford, Inorganic Chemistry Laboratory, South Parks Road, Oxford OX1 3QR, UK \\ ${ }^{3}$ Department of Chemistry, University of Oxford, Parks Road, Oxford OX1 3QR, UK
}

(Dated: November 4, 2019)

\begin{abstract}
$\mathrm{CaKFe}_{4} \mathrm{As}_{4}$ (1144) is a unique stoichiometric iron-based superconductor which harbours high upper critical fields and large critical current densities. In this work, we describe a study to optimize the synthesis conditions of stoichiometric polycrystalline samples of $\mathrm{CaKFe}_{4} \mathrm{As}_{4}$ and asses their structural, magnetic and transport properties. The samples were prepared over a wide temperature range $\left(900-1100^{\circ} \mathrm{C}\right)$ and the pure phase formation is centered around $955^{\circ} \mathrm{C}$. Outside this temperature region, impurity phases of $\mathrm{KFe}_{2} \mathrm{As}_{2}$ and $\mathrm{CaFe}_{2} \mathrm{As}_{2}$ can also form. Magnetic susceptibility and resistivity measurements establish that the critical temperature reaches $\sim 34$ $\mathrm{K}$ for the optimum synthesis conditions and the critical current reaches $2 \times 10^{4} \mathrm{~A}-\mathrm{cm}^{-2}$. The post-annealing process demonstrates the stability of the 1144 phase up to $500^{\circ} \mathrm{C}$, however, under higher temperature annealing, phase degradation occurs. Our study indicates that the formation of phase-pure 1144 occurs over a much narrower window and its highly prone to multi-phase formation as compared with the 122 family. As a result, the superconducting properties are enhanced for the pure 1144 phase but they are likely to be affected by the interand intra-granular behaviour originating from the microstructural nature of polycrystalline $\mathrm{CaKFe}_{4} \mathrm{As}_{4}$, similar to other iron-based superconductors. Based on our study, we construct the phase diagram for polycrystalline 1144 and compared it with that reported for 1144 single crystal.
\end{abstract}

\section{INTRODUCTION}

Iron based superconductors are exciting multiband electronic systems that display complex physics and an unique pairing mechanism in different crystallographic families ${ }^{1}$. Furthermore, they show great potential for practical applications due to their high transition temperature up to $58 \mathrm{~K}^{2}$, extremely high upper critical field $\left(\mu_{0} H_{c 2} \sim 100 \mathrm{~T}\right)$, very low anisotropy and rather large critical current density $\left(J_{c}\right.$ $\sim 10^{6}-10^{8} \mathrm{~A} / \mathrm{cm}^{2}$ ) with independent of the field at low temperatures ${ }^{3-5}$. Among various families of iron-based superconductors, the 122 family $\left(\mathrm{AEFe}_{2} \mathrm{As}_{2}, \mathrm{AE}=\mathrm{Ba}, \mathrm{Sr}\right)$ was believed to be suitable for applications due to its excellent superconducting properties, stable pure phases obtained by an easy synthesis process and an advantageous grain-boundary nature ${ }^{4,5}$. Significant research efforts have been directed toward fabrication of superconducting 122 wires/tapes ${ }^{5}$. However, to archive optimum superconductivity, the materials are chemically doped and the highest superconducting temperature of $38 \mathrm{~K}$ was reported for $\mathrm{K}$ doping $^{6}$, but it still remains a challenge to control of the actual doping level of these materials.

To overcome this issue, a new family 1144 of stoichiometric iron-based superconductors, $\mathrm{AeAFe}_{4} \mathrm{As}_{4}(\mathrm{Ae}=\mathrm{Ca}, \mathrm{Sr}$ and $\mathrm{A}=\mathrm{K}, \mathrm{Rb}, \mathrm{Cs}$ ) were discovered with critical temperature, $T_{c}$, between 31 and $36 \mathrm{~K}$ without the need for additional doping ${ }^{7,8}$. Recent studies on 1144 single crystals ${ }^{3,8,9}$ reveal highly improved superconducting properties as compared with those reported for the 122 family ${ }^{10,11}$. These include a high critical current density of the order of $10^{8} \mathrm{~A} / \mathrm{cm}^{2}$ at low temperature, a rather robust field dependence as well as an isotropic upper critical field of up to $\mu_{0} H_{c 2} \simeq 100 \mathrm{~T}$. These experimental findings place the 1144 family as a strong contender for magnet application at around $20 \mathrm{~K}$, where the niobium based superconductors ${ }^{12}$ cannot play a role owing to their lower $T_{c}$.
The fabrication of superconducting tapes and wires for bulk applications requires the development of high quality polycrystalline (powder) materials. Iyo et $a l^{7}$ synthesized powder $\mathrm{CaKFe}_{4} \mathrm{As}_{4}$ but found that these samples can form also the impurity phases of $\mathrm{CaFe}_{2} \mathrm{As}_{2}$ and $\mathrm{KFe}_{2} \mathrm{As}_{2}$. Furthermore, single crystal growth phase diagram of $\mathrm{CaKFe}_{4} \mathrm{As}_{4}{ }^{13}$ shows that the 1144 phase can be only formed in the temperature range between $920^{\circ} \mathrm{C}$ to $980^{\circ} \mathrm{C}$ and has a strong sensitivity to impurity phase formation outside this range ${ }^{13}$. For the wires and tapes fabrication, a robust synthesis process is needed that can produce very high quality and large amount of the 1144 precursor powders. Therefore, it is important to optimize the synthesis of powder $\mathrm{CaKFe}_{4} \mathrm{As}_{4}$ for revealing its intrinsic superconducting properties. Recently $\mathrm{CaKFe}_{4} \mathrm{As}_{4}$ tapes and wires ${ }^{14,15}$ have been produced with a critical current density of $10^{4} \mathrm{~A} / \mathrm{cm}^{2}$ at $4.2 \mathrm{~K}$, despite a large amount of impurities. This could be increased dramatically if the powder synthesis process is improved.

In this work, we present a detailed study of superconducting properties of stoichiometric $\mathrm{CaKFe}_{4} \mathrm{As}_{4}$ in polycrystalline form. We use a broad range of synthesis temperatures to optimize the growth process, similar to those used for the single crystal ${ }^{13}$. We performed a series of physical properties studies to asses the structure, microstructure, magnetization and resistivity of different batches of these samples. Different types of characterization suggest that synthesis temperature of $955^{\circ} \mathrm{C}$ for $6 \mathrm{~h}\left(T_{\text {synth }}^{\mathrm{opt}}\right)$ is the optimum condition for synthesizing phase pure polycrystalline $\mathrm{CaKFe}_{4} \mathrm{As}_{4}$ with highest onset $T_{c}$ value of $34.2 \mathrm{~K}$ with a sharp transition width $\Delta T_{c} \simeq 2 \mathrm{~K}$. The superconducting properties of the best samples are characterised further by different measurements and are found to show behaviour similar to those reported for single crystals of $\mathrm{CaKFe}_{4} \mathrm{As}_{4}{ }^{13}$. 


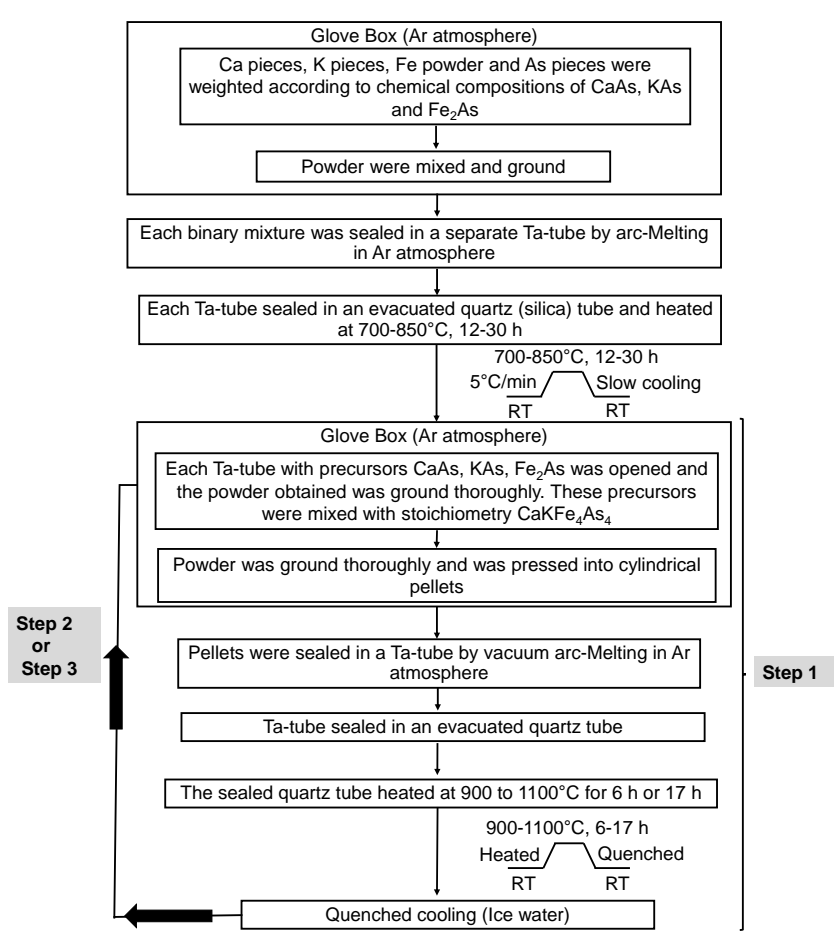

FIG. 1. Synthesis block diagram followed for each set of polycrystalline samples of $\mathrm{CaKFe}_{4} \mathrm{As}_{4}$ (1144) prepared at different synthesis temperatures, $T_{\text {synth }}$, as described in detail in the text.

\section{EXPERIMENTAL DETAILS}

In order to optimize the growth conditions, a series of polycrystalline samples of $\mathrm{CaKFe}_{4} \mathrm{As}_{4}$ were prepared at different synthesis temperature, $T_{\text {synth }}$, varying between $900^{\circ} \mathrm{C}$ and $1100^{\circ} \mathrm{C}$ following the block diagram shown in Figure 1. High purity starting reagents: Ca chunks (99.99\%), Fe powder (99.99\%), K (99.99\%) and As (99.99\%) pieces were used. Firstly, precursors CaAs and KAs were prepared by placing stoichiometric amounts of $\mathrm{Ca}$ or $\mathrm{K}$ and $\mathrm{As}$ in a Ta tube, then welded sealed Ta tube in Ar atmosphere and then sealed in an evacuated quartz (silica) tube. The sealed tube is kept at $860^{\circ} \mathrm{C}$ for $30 \mathrm{~h}$ for CaAs and at $650^{\circ} \mathrm{C}$ for $12 \mathrm{~h}$ for KAs. A similar method was used to prepare FeAs by heating Fe powder and As powder at $700^{\circ} \mathrm{C}$ for $12 \mathrm{~h}$. For the synthesis of the 1144 powder, the starting precursors were mixed according to a stoichiometric $\mathrm{CaKFe}_{4} \mathrm{As}_{4}$ ratio in an agate mortar, then placed into a Ta tube. Other possible crucible materials, such as quartz tube and $\mathrm{Nb}$ have not been used in order to minimize the possible side reactions of $\mathrm{Ca}, \mathrm{K}$ and As, respectively. The Ta tube was clamped closed by pliers and then sealed by welding in an Ar atmosphere to prevent any oxidation. Finally, the Ta tube was inserted in an evacuated quartz tube, sealed and placed inside a box furnace and kept at $T_{\text {synth }}$ for $6 \mathrm{~h}$ for each sample. The resultant powder was ground, pressed into pellets by applying a uniaxial pressure of $3 \mathrm{MPa}$, sealed into a fresh Ta tube which was sealed in an evacuated quartz tube and then heated at the same synthesis temperature $T_{\text {synth }}$ for $6 \mathrm{~h}$ (step 2 in Figure 1). After this reaction, the obtained pellets were brittle. Hence, the synthesized pellets were reground and the powder was pressed by applying a pressure of $3 \mathrm{MPa}$. These pellets were again placed in Ta tube that was sealed afterwards in Ar atmosphere. Finally, this Ta tube was inserted in an evacuated quartz tube and placed in a furnace at same $T_{\text {synth }}$ for $2 \mathrm{~h}$ (step 3, Figure 1). It is worth to note that for each step the furnace was heated up to $T_{\text {synth }}$ before placing the sample inside. At the end of each step, the sample was taken out of the furnace at $T_{\text {synth }}$ and quenched into ice water. These procedures are very important to avoid the formation of 122 phase containing $\mathrm{KFe}_{2} \mathrm{As}_{2}$ or $\mathrm{CaFe}_{2} \mathrm{As}_{2}$. Once we had identified the optimum temperature for the phase pure sample, we further optimized this phase by post annealing. All the powder handling procedures were done inside the glove box. Powder X-ray diffraction patterns were used to characterize the resulting bulk samples within the $2 \theta$ angular range from 5 to $80^{\circ}$ and the data were collected in Bragg-Brentano reflection geometry with $\mathrm{Cu}-\mathrm{K} \alpha$ radiation. The samples were stable in air, however, they were prepared inside glove box by using a home designed sealed holder for $\mathrm{X}$-ray measurements to protect against possible degradation in air. The profile analysis and lattice parameters were obtained from Rietveld refinements on the XRD data employing the Fullprof software suite ${ }^{16}$. Microstructural characterizations were performed using a field-emission scanning electron microscope (FESEM). Magnetic measurements up to $16 \mathrm{~T}$ were performed using Vibrating Sample Magnetometer (VSM) implemented in a Quantum Design PPMS in temperature range between 2 to $50 \mathrm{~K}$ under zero-field and field-cooling conditions. The zero-field cooled (ZFC) magnetization was measured by cooling the bulk sample under zero field to $2 \mathrm{~K}$, then the magnetic field was applied and the data were collected during the warming up process. For field-cooled (FC) mode, the sample was cooled down to $2 \mathrm{~K}$ in preferred field and the data was recorded in heating cycle in presence of the same applied field. The temperature dependence of the resistivity in zero magnetic field was measured in a home-made flow cryostat in the temperature range 4.2 to $300 \mathrm{~K}$ and the magnetoresistance was measured up to $16 \mathrm{~T}$. The electrical contacts were made using thin gold wires with the help of silver epoxy. The upper critical field, $H_{c 2}$, was extracted both from the temperature dependence of magnetization and resistivity in the temperature range of 2 to $40 \mathrm{~K}$ and different constant magnetic fields.

\section{RESULTS AND DISCUSSION}

\subsection{XRD and microstructural analysis}

Powder X-ray diffraction patterns were recorded for each set of samples synthesized at different $T_{\text {synth }}$, as shown in Figure 2(a). $\mathrm{CaKFe}_{4} \mathrm{As}_{4}$ crystallises in $P 4 / \mathrm{mmm}$ space group with $\mathrm{Ca}$ and $\mathrm{K}$ ordering on distinct crystallographic sites, differing from the $14 / \mathrm{mmm}$ structure of $\mathrm{Ca}_{0.5} \mathrm{Na}_{0.5} \mathrm{Fe}_{2} \mathrm{As}_{2}$ in which the $\mathrm{Ca}$ and $\mathrm{Na}$ share a single crystallographic site ${ }^{7,13}$. 

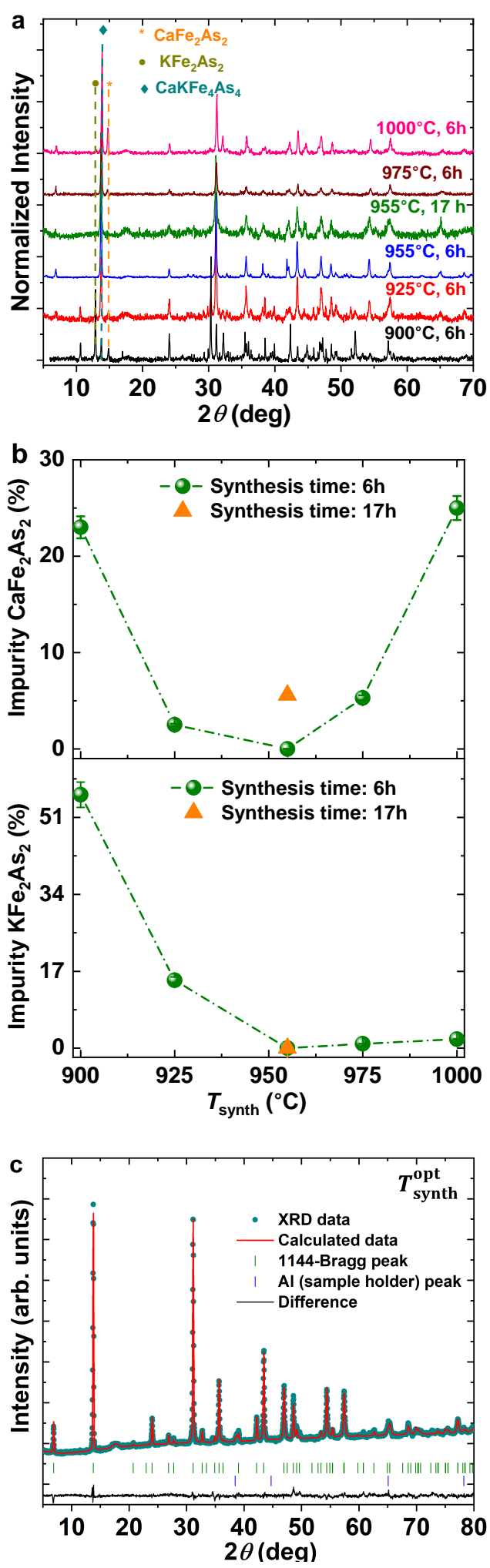

FIG. 2. (a) Powder x-ray diffraction patterns (XRD) of $\mathrm{CaKFe}_{4} \mathrm{As}_{4}$ prepared at different synthesis temperatures, $T_{\text {synth }}$. (b) Temperature dependence of the impurity phases $\mathrm{CaFe}_{2} \mathrm{As}_{2}$ and $\mathrm{KFe}_{2} \mathrm{As}_{2}$ (122) estimated as the ratio between the main diffraction peak of the 122 and 1144 phases. (c) Experimental and calculated diffraction patterns and their difference for the room temperature X-ray diffraction data of $\mathrm{CaKFe}_{4} \mathrm{As}_{4}$ prepared at optimum synthesis conditions $\left(T_{\mathrm{synth}}^{\mathrm{opt}}\right)$.
The presence of the 001 reflection at a d-spacing of $\sim 12.9 \AA$ ( $\sim 6.9$ degrees two-theta for $\mathrm{Cu}-\mathrm{K} \alpha$ radiation) is indicative of whether the $\mathrm{Ca}$ and $\mathrm{K}$ are crystallographically ordered. The synthesised compounds are well fitted with the ordered structure in space group $P 4 / \mathrm{mmm}$, but additional impurity phases occur in some samples. We have estimated the concentration and nature of the impurity phases by using the comparative intensity of the different phases, as shown in Figure 2(b). We find that the sample prepared at $900^{\circ} \mathrm{C}$ is dominated by impurity phases formed mainly of $\mathrm{KFe}_{2} \mathrm{As}_{2}(\sim 50 \%)$ and $\mathrm{CaFe}_{2} \mathrm{As}_{2}(\sim 25 \%)$. With increasing $T_{\text {synth }}$, both impurity phases decreased significantly and no impurity phase is detected in the $\mathrm{CaKFe}_{4} \mathrm{As}_{4}$ synthesized at $955^{\circ} \mathrm{C}$ for $6 \mathrm{~h}$. For higher $T_{\text {synth }}$, the 1144 phase coexists with a dominant impurity phase $\mathrm{CaFe}_{2} \mathrm{As}_{2}$ evident in the diffraction pattern $(\sim 25 \%$ for the growth at $\left.1000^{\circ} \mathrm{C}\right)$. A small amount $(\sim 1-2 \%)$ of $\mathrm{KFe}_{2} \mathrm{As}_{2}$ is also observed in the diffractogram (as shown in Figure 2(b)). Furthermore, we have observed that samples prepared at $955^{\circ} \mathrm{C}$ for a longer period of time of $17 \mathrm{~h}$ have the tendency to start forming $\mathrm{CaFe}_{2} \mathrm{As}_{2}$ impurity phases, as shown in Figure 2(a) and (b). These studies suggest that the pure phase of polycrystalline samples of $\mathrm{CaKFe}_{4} \mathrm{As}_{4}$ could be formed in a very narrow temperature window and for a short heating duration i.e. $955^{\circ} \mathrm{C}$ for $6 \mathrm{~h}\left(T_{\text {synth }}^{\text {opt }}\right)$. This result is similar to the optimized temperature for the phase pure single crystals of $\mathrm{CaKFe}_{4} \mathrm{As}_{4}$ which is lying between 920 to $980^{\circ} \mathrm{C}^{13}$.

Figure 2(c) shows the measured and Rietveld-fitted XRD patterns of the 1144 sample prepared in optimum conditions at $T_{\mathrm{synth}}^{\mathrm{opt}}$. The refined lattice parameters for different samples are listed in the Table I and the lattice parameters vary slightly between the samples imply that there is a subtle structural difference in their compositions. However, there is no trend in the lattice parameter variation and it should be noted that the samples synthesized at non-optimal temperatures possess impurity phases, and thus these small variations may be the result of the large error bar in the refinement. The lattice parameters for the optimum samples grown at $955^{\circ} \mathrm{C}$ are comparable to that of the reported single crystals ${ }^{8,13}$. Additionally, the energy dispersive X-ray (EDAX) technique found for the optimum sample that the molar ratio $\mathrm{Ca}: \mathrm{K}: \mathrm{Fe}: \mathrm{As}$ was 1:0.99:4.1:3.98. This is consistent with a stoichiometric $\mathrm{CaKFe}_{4} \mathrm{As}_{4}$ composition and similar to other reports based on 1144 single crystals ${ }^{8,13}$.

In order to investigate the local chemical composition, the morphology and grain boundaries of the polycrystalline 1144 samples prepared by the optimum conditions ( $\left.T_{\mathrm{synth}}^{\mathrm{opt}}\right)$ we used backscattered scanning electron microscopy (BSE-SEM) as shown in the images in the Figure 3(a)-(d). The sample surface was carefully polished by using sand papers without lubricants. The crystallographic density from lattice parameters and the calculated density from sample dimensions for our optimal 1144 sample is $5.22 \mathrm{~g} / \mathrm{cm}^{3}$ which is similar to the reported value $e^{7,13}$. However, the sample has a denser micro-structure and microscale pores appeared along many grain boundaries as shown by the light gray and black contrasts corresponding to the 1144 phase and pores, respectively. We have not observed any micro-cracks between the grains, 
TABLE I. Lattice parameters and the goodness of fit expressed by $R_{\mathrm{Bragg}}$ of different samples of $\mathrm{CaKFe}_{4} \mathrm{As}_{4}(1144)$ prepared at various synthesis temperatures, $T_{\text {synth }}$.

\begin{tabular}{llll}
\hline$T_{\text {synth }}\left({ }^{\circ} \mathrm{C}\right)$ & $a(\AA)$ & $c(\AA)$ & $R_{\text {Bragg }}$ \\
\hline $925^{\circ} \mathrm{C}, 6 \mathrm{~h}$ & $3.8657(4)$ & $12.893(5)$ & 2.61 \\
\hline $955^{\circ} \mathrm{C}, 6 \mathrm{~h}\left(T_{\text {synth }}^{\text {opt }}\right)$ & $3.8673(1)$ & $12.841(1)$ & 1.35 \\
\hline $955^{\circ} \mathrm{C}, 17 \mathrm{~h}$ & $3.8644(5)$ & $12.893(5)$ & 1.46 \\
\hline $975^{\circ} \mathrm{C}, 6 \mathrm{~h}$ & $3.8683(3)$ & $12.853(3)$ & 3.77 \\
\hline $1000^{\circ} \mathrm{C}, 6 \mathrm{~h}$ & $3.8673(4)$ & $12.842(3)$ & 3.65 \\
\hline $600^{\circ} \mathrm{C}$ post-annealed, $1 \mathrm{~h}$ & $3.8615(2)$ & $12.883(2)$ & 3.04 \\
\hline $900^{\circ} \mathrm{C}$ post-annealed, $1 \mathrm{~h}$ & $3.8544(4)$ & $12.862(5)$ & 3.07 \\
\hline
\end{tabular}
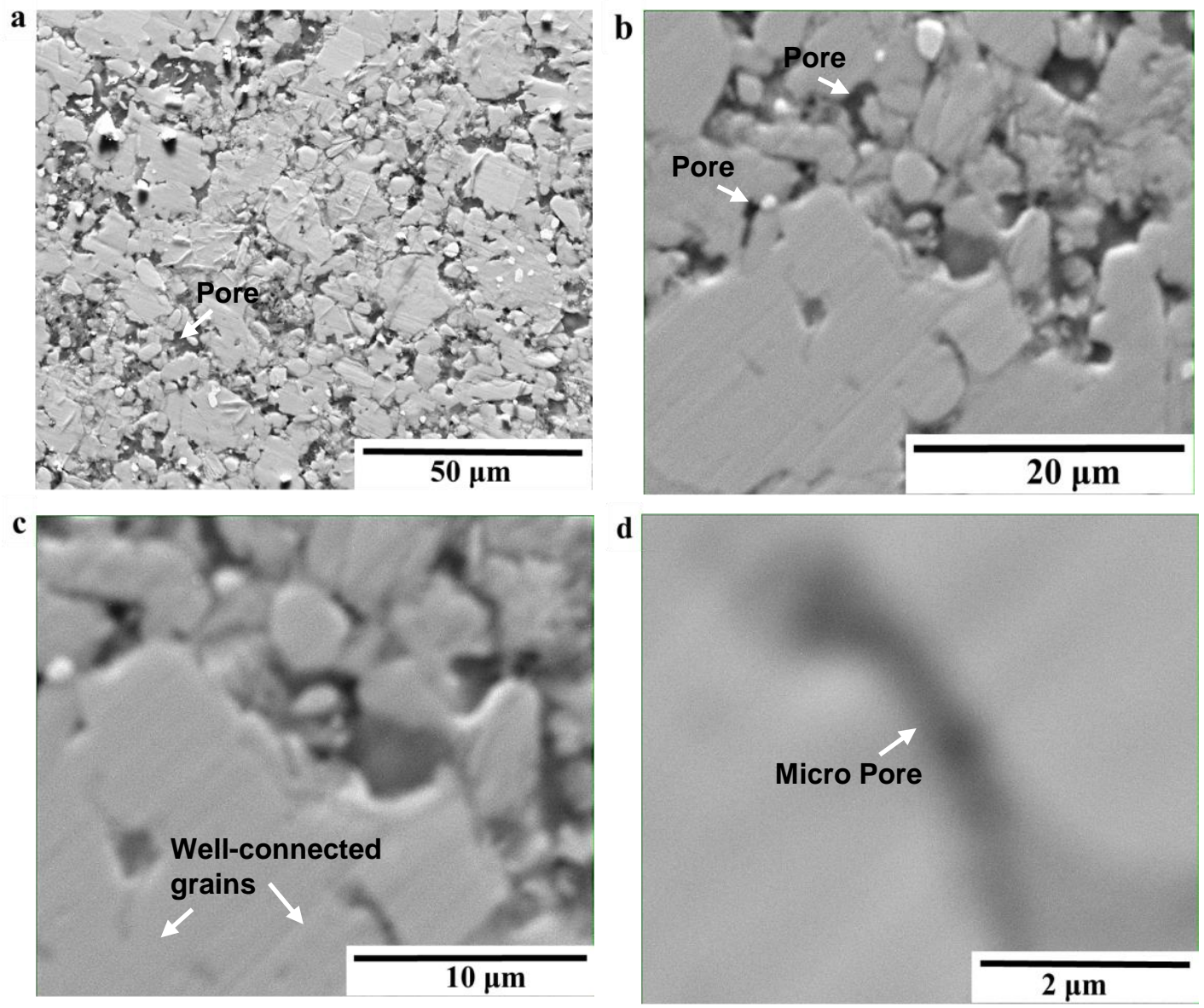

FIG. 3. (a)-(d) Back-scattered electron SEM images of the polycrystalline $\mathrm{CaKFe}_{4} \mathrm{As}_{4}$ (1144) sample prepared at $T_{\mathrm{synth}}^{\mathrm{opt}}$. Light gray and black contrasts correspond to 1144 and pores respectively. In (a)-(b) no cracks are observed and the slightly white contrast could be related to small deviations from the $\mathrm{CaKFe}_{4} \mathrm{As}_{4}$ stoichiometry during polishing.

as found in other iron-based superconductors like REFeAsO (RE1111, RE = rare earth) and 122 where extensive cracking appears either at the grain boundaries or within grains ${ }^{5,17-19}$. Images in Figure 3(c)-(d) revealed a disk-like shape of grains with an average size of 3-4 $\mu \mathrm{m}$, quite clean grain boundaries and many well-connected grain boundaries. The superconducting properties are likely to be affected by the presence of larger pores that would lead to poorer connections between the corresponding grains as compared with the samples containing well-connected grains. Future studies could improve the grain connectivity using other techniques, such as hot or cold pressing or high pressure synthesis method ${ }^{5}$. In other iron-based superconductors, slow cooling generally helps the grain connectivity ${ }^{2,20}$. To address this issue, we have slowly 
cooled a sample from $955^{\circ} \mathrm{C}$ and we observe that already below $940^{\circ} \mathrm{C}$ the impurity phases of $\mathrm{CaFe}_{2} \mathrm{As}_{2}$ and $\mathrm{KFe}_{2} \mathrm{As}_{2}$ had started to appear. This was also confirmed by the XRD data analysis which suggests that 1144 can be prepared only in a very narrow temperature region, similar to the single crystal growth $^{13}$. The microstructure of the 1144 samples prepared at different $T_{\text {synth }}$ indicate many white spots in BSE images and EDX at these spots confirmed the presence of oxygen which indicates that the impurity phases $\mathrm{CaFe}_{2} \mathrm{As}_{2}$ and $\mathrm{KFe}_{2} \mathrm{As}_{2}$ are not stable in air. During polishing the surface of these samples, $\mathrm{CaFe}_{2} \mathrm{As}_{2}$ and $\mathrm{KFe}_{2} \mathrm{As}_{2}$ could react with moisture or air and these 122 phases are not very stable in air, as reported in other studies $^{21,22}$.

\subsection{Magnetic property measurements}

To assess the superconducting properties of the $\mathrm{CaKFe}_{4} \mathrm{As}_{4}$ polycrystalline samples prepared at different $T_{\text {synth }}$ we measure the temperature dependence of the magnetic susceptibility both zero-field-cooled (ZFC) and field-cooled (FC) conditions in $\mu_{0} H=20 \mathrm{Oe}$, as shown in Figure 4(a). Here, the magnetic susceptibility is corrected for the demagnetization factor, as described in Ref. 3 and 23. Perfect diamagnetic susceptibility is obtained for the optimum sample ( $\left.T_{\mathrm{synth}}^{\mathrm{opt}}\right)$ shown in Figure 4(b)-(c) which has the highest transition temperature of $33.2 \mathrm{~K}$ and a sharp transition, similar to other reports ${ }^{7,8}$. The variation of transition temperature with respect to the synthesis temperature is shown in Figure 4(c) which clearly depicts a high $T_{c}$ for the optimum synthesis conditions similar to XRD data. By increasing the $T_{\text {synth }}$ above $955^{\circ} \mathrm{C}$, the superconducting transition decreased very rapidly and the transition to superconducting state broadens. Furthermore, for the sample prepared at $955^{\circ} \mathrm{C}$ for longer time $(17 \mathrm{~h})$, the transition temperature is almost similar to the optimum sample but the superconducting volume fraction is reduced. This may be caused by a small amount of the impurity phases as observed from the XRD data in Figure 2(a). On the other hand, the sample prepared at $900^{\circ} \mathrm{C}$ shows a broad jump near $160 \mathrm{~K}$ due to the magnetic-structural transition of non-superconducting phase $\mathrm{CaFe}_{2} \mathrm{As}_{2}{ }^{11}$ and a huge paramagnetic background is also observed due to the presence of other impurity phases (not shown here). As the synthesis temperature is increased, the samples heated at $925^{\circ} \mathrm{C}$ exhibits a marked drop at $30.3 \mathrm{~K}$ in both $\mathrm{ZFC}$ and FC curves, indicating the occurrence of superconductivity. However, the transition is very broad with a relatively low superconducting volume fraction suggesting the presence of the 1144 phase together with other phases, as discussed in the previous sections.

\subsection{Transport properties}

Figure 5(a) shows the temperature dependence of the electrical resistivity for the polycrystalline 1144 samples synthesized at various temperatures $T_{\text {synth }}$. The data reveal a metallic normal state for all samples, similar to single crystal studies ${ }^{3,8}$. The sample prepared at $900^{\circ} \mathrm{C}$ does not shows
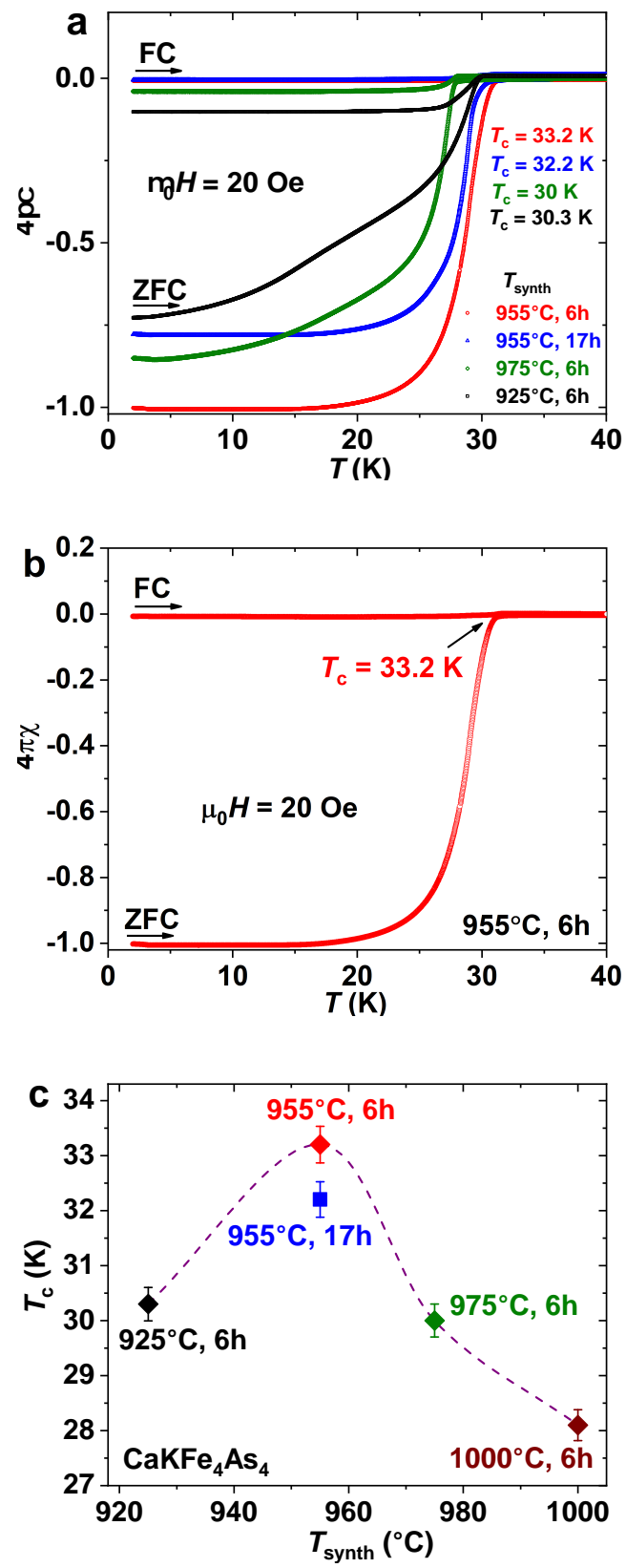

FIG. 4. (a) The temperature dependence of the magnetic susceptibility of $\mathrm{CaKFe}_{4} \mathrm{As}_{4}$ prepared at different synthesis temperature with zero-field-cooling (ZFC) and field-cooling (FC) in magnetic field of $\mu_{0} H=20$ Oe. (b) ZFC and FC magnetization in 20 Oe for the sample prepared at $T_{\mathrm{synth}}^{\mathrm{opt}}$. (c) The dependence of critical temperature, $T_{c}$, versus the synthesis temperature, $T_{\mathrm{synth}}$, obtained from the magnetization data.

the superconducting transition but has a broad jump in the resistivity near $160 \mathrm{~K}$ that might be due to the structural transition of the impurity phase, $\mathrm{CaFe}_{2} \mathrm{As}_{2}{ }^{24}$. For other samples, the resistivity decreases monotonically with decreasing temperature and the onset of superconducting transition occurs between 32 to $34 \mathrm{~K}$ which is slightly higher than that from the 

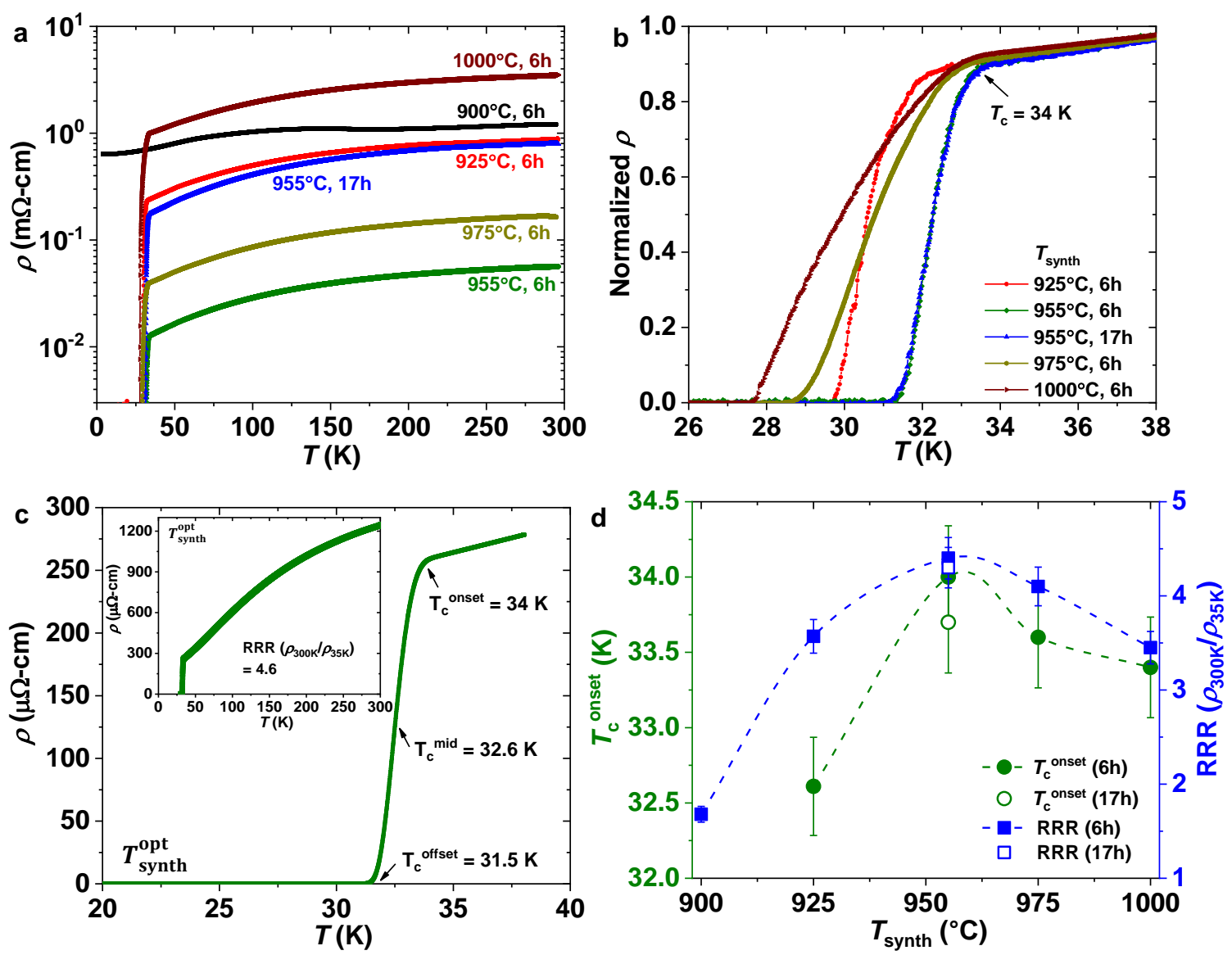

FIG. 5. (a) Temperature dependence of the resistivity, $\rho$, between 4 to $300 \mathrm{~K}$ for the polycrystalline $\mathrm{CaKFe}_{4} \mathrm{As}_{4}$ samples prepared at different $T_{\text {synth. }}$ (b) Low-temperature superconducting region of the normalized resistivity by the room temperature resistivity value is shown for all samples. (c) Low-temperature resistivity for the $\mathrm{CaKFe}_{4} \mathrm{As}_{4}$ sample prepared at $T_{\mathrm{synth}}^{\mathrm{opt}}$. The inset shows the resistivity up to room temperature for the same sample. (d) The evolution of the onset transition temperature, $T_{c}^{\text {onset }}$, and the residual resistivity ratio, RRR $=\rho_{300 K} / \rho_{35 K}$, with the synthesis temperature, $T_{\text {synth }}$.

magnetization study shown in Figure 4, due to the percolative effects present in transport. The resistivity values of different samples vary with $T_{\text {synth }}$, as shown in Figure 5(d), where higher resistivity suggest a larger concentration of impurity phases, as mentioned in the XRD section. To compare different samples, the resistivity data are normalized with respect to the room temperature, as shown in Figure 5(b). We find that the sample synthesized in optimum conditions $\left(T_{\mathrm{synth}}^{\mathrm{opt}}\right)$ is the most conducting having the lowest resistivity, as compared to the other samples. It is worth to mention that its resistivity is smaller than that of polycrystalline $\mathrm{CaKFe}_{4} \mathrm{As}_{4}$ samples in previously reports ${ }^{7}$. The remarkable reduction of the resistivity of the best 1144 sample is an evidence for a much better grain connectivity compared to other samples.

The onset transition temperature, $T_{c}^{\text {onset }}$, and the residual resistivity ratio, RRR $=\rho_{300 K} / \rho_{35 K}$, of all samples are plotted in Figure 5(d). The $T_{c}^{\text {onset }}$ reaches the maximum value of $34 \mathrm{~K}$ and it has a sharp transition for the sample prepared at $T_{\mathrm{synth}}^{\mathrm{opt}}$ as shown in Figure 5(c). This is a clear indication of higher homogeneity, better grain connectivity and a phase pure sample. On the other hand, for the samples grown in different synthesis conditions, $T_{c}$ is always lower, the transition width, $\Delta T_{c}=T_{c}^{\text {onset }}-T_{c}^{\text {offset }}$, is wider and the resistivity value at the room temperature $\left(\rho_{300 K}\right)$ decrease with increasing synthesis temperature, $T_{\text {synth }}$. The maximum of RRR and the minimum of $\Delta T_{c}$ for the optimum grown sample are another transport signature of the high quality of this polycrystalline $\mathrm{CaKFe}_{4} \mathrm{As}_{4}$ bulk sample. Note that the RRR is 4.6 for our best sample, almost factor three smaller than the value reported for the 1144 single crystal ${ }^{3,8}$. Furthermore, the obtained $T_{c}$ and RRR for the optimal 1144 polycrystalline have similar values to that reported for the 1144 powder by Iyo et $a .^{7}$ ( $T_{c} \sim 33 \mathrm{~K}$ and $\mathrm{RRR} \sim 7$ ), which has higher resistivity value ( $\sim 3.5$ milliohm-cm at $300 \mathrm{~K})$ and low superconducting volume fraction $(\sim 80 \%)$.

Next we discuss the upper critical field $H_{c 2}$ obtained from 

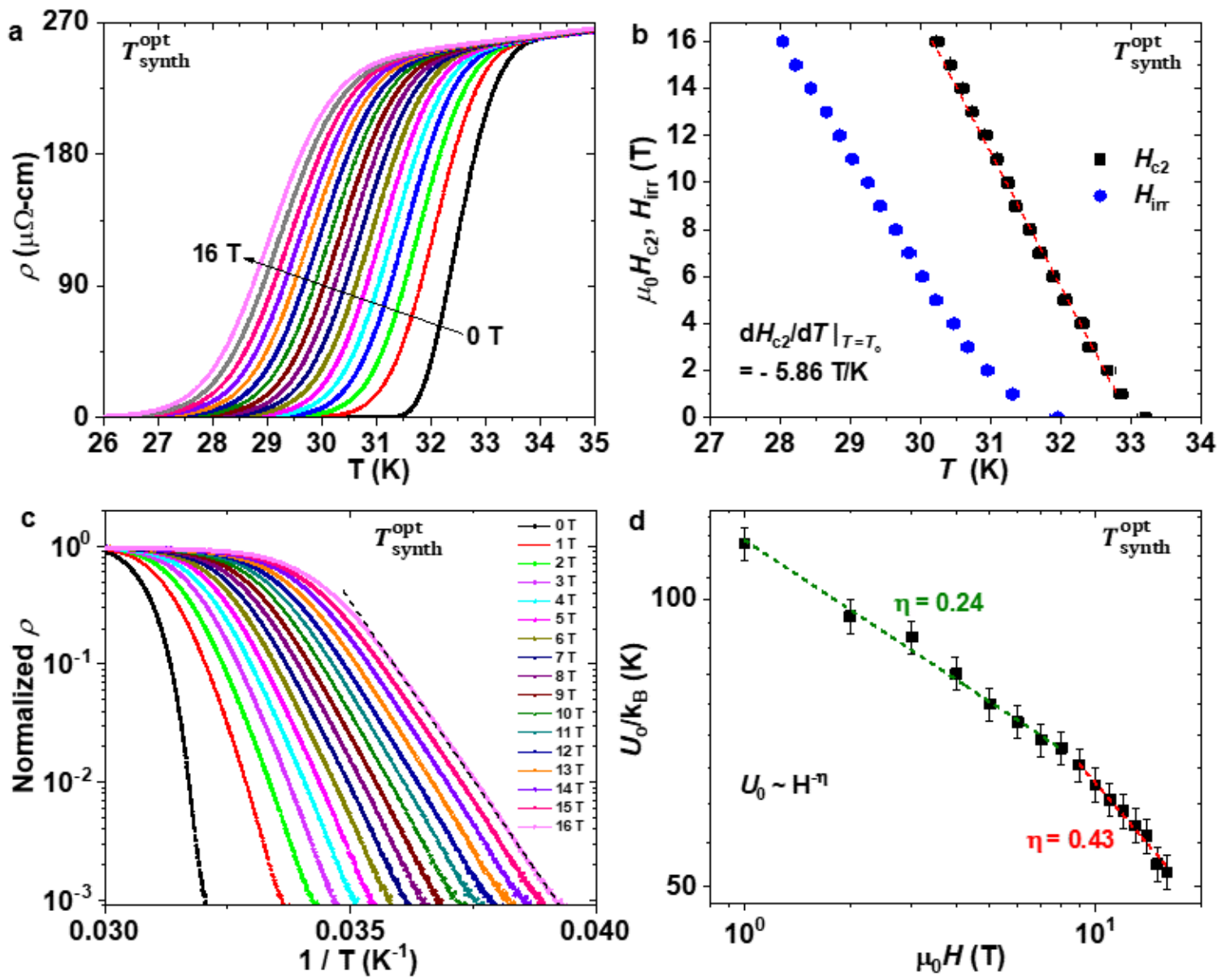

FIG. 6. (a) Temperature dependence on the resistivity measured is different magnetic fields for the best sample of $\mathrm{CaKFe}_{4} \mathrm{As}_{4}$ prepared at $T_{\mathrm{synth}}^{\mathrm{opt}}$. (b) The upper critical field, $H_{c 2}$ (close rectangle) and the irreversibility field, $H_{i r r}$, (close circle) of the same sample is determined as the $90 \%$ and $10 \%$ of the normal state resistivity, respectively. (c) Arrhenius plot of the electrical resistivity of $\mathrm{CaKFe}_{4} \mathrm{As}_{4}$ in the presence of various magnetic fields for the same sample. Black dashed lines show linear fits in the low temperature regime, reflecting thermal activation behaviour. (d) The field dependence of the activation energy $\left(U_{0} / k_{B}\right)$ for $\mathrm{CaKFe}_{4} \mathrm{As}_{4}$ prepared at $T_{\mathrm{synth}}^{\mathrm{opt}}$. The red dashed line shows the fitting according to a power law dependence, $U_{0} \sim H^{-\eta}$.

magnetotransport studies for our best polycrystalline sample grown in optimum conditions $\left(T_{\text {synth }}^{\text {opt }}\right)$. Figure 6(a) shows the temperature dependence of the resistivity under various magnetic fields up to $16 \mathrm{~T}$ and the magnetic field is applied perpendicular to the direction of the current flow. The resistivity curve shows a sharp transition at zero magnetic field, indicating that grains maintain a good percolation path between them. The onset transition and zero resistance temperature exhibit a gradual shift to lower temperatures with increasing magnetic field. We estimate the upper critical magnetic field, $H_{c 2}$, and the irreversibility field, $H_{i r r}$, respectively as the magnetic field at which the resistivity reaches $90 \%$ and $10 \%$ of the normal state resistivity, $\rho_{n}$, respectively. Based on these parameters we construct the $H-T$ phase diagram for the polycrystalline $\mathrm{CaKFe}_{4} \mathrm{As}_{4}$, as shown in Figure 6(b). Furthermore, we can estimate the zero-temperature upper critical field $H_{c 2}(0)$ using the Werthamer-Helfand-Hohenberg
(WHH) formula ${ }^{25}: \mu_{0} H_{c 2}=-0.693 T_{c}\left(\mathrm{~d} H_{c 2} / \mathrm{d} T\right)_{T \rightarrow T_{c}}$. The slope of the upper critical field $\left(\mu_{0} d H_{c 2} / d T\right)$ is estimated from the $H_{c 2}$ versus $T$ plots, and the value is $-5.86 \mathrm{~T} \mathrm{~K}^{-1}$. Using transition temperature $T_{c}=34 \mathrm{~K}$, we find $\mu_{0} H_{c 2}(0)=$ $138 \mathrm{~T}$, similar to the reported value for the single crystal $^{3,8}$. Also, the calculated irreversibility field $\mu_{0} H_{\text {irr }}(0)$ is estimated as $100 \mathrm{~T}$ for a high quality $\mathrm{CaKFe}_{4} \mathrm{As}_{4}$ polycrystalline sample. This obtained $H_{c 2}$ value of polycrystalline 1144 from this magneto-resistance study is almost a factor of 2 smaller than that of the previous report from the magnetization measurements ${ }^{3}$. It suggests that grain boundaries or pores increase the density of the pinning centers to give rise to large upper critical fields during the magnetization measurements of the polycrystalline 1144 sample. From the upper critical field $H_{c 2}$ one can estimate the coherence length, $\xi$, using $H_{c 2}$ $=\phi_{0} /\left(2 \pi \xi^{2}\right)$, where $\phi_{0}$ is the flux quantum. The coherence length is estimated to be $1.5 \mathrm{~nm}$ for $\mathrm{CaKFe}_{4} \mathrm{As}_{4}$, similar to 
the reported value for a single crystal ${ }^{3,8}$. Furthermore, this $H_{c 2}$ value of this stoichiometric $\mathrm{CaKFe}_{4} \mathrm{As}_{4}$ superconductor is much higher than other stoichiometric iron-based superconductors, such as $\mathrm{LiFeAs}^{26}$.

The broadening of the resistive transitions with increasing magnetic fields can be explained in terms of energy dissipation due to vortex motion in the mixed state. Energy is dissipated whenever vortices move ${ }^{27}$. Traditionally, one can distinguish two regimes of dissipation in the mixed state: flux creep when the pinning force dominates and flux flow when the Lorentz force dominates. The resistive broadening is generally due to the creep of vortices which are thermally activated. The resistivity in the thermally activated flux flow (TAFF) regime of the flux creep is given by an Arrhenius equation $\rho=\rho_{0} \exp \left[-U_{0} / \mathrm{k}_{\mathrm{B}} T\right]$, where $\rho_{0}$ is the field independent pre-exponential factor that is normal state resistivity above the $T_{c}, \mathrm{k}_{\mathrm{B}}$ is Boltzmann's constant and $U_{0}$ is the TAFF activation energy. To reveal the origin of thermally activated $\rho(T, B)$, we present the Arrhenius plots of representative low resistive data as shown in Figure 6(c), where the resistivity is plotted on a logarithmic scale $(\log \rho)$ versus the inverse of temperature $\left(T^{-1}\right)$. From the Arrhenius plots below $T_{c}$ we extract the activation energy $U_{0}$, related to the slope of each curve with respect to field. Figure 6(d) shows the magnetic field dependence of the activation energy, $U_{0}$, of the optimum $\mathrm{CaKFe}_{4} \mathrm{As}_{4}$ sample and it displays two different regimes in magnetic fields up to $16 \mathrm{~T}$. The activation energy varies as $H^{-0.24}$ up to $7 \mathrm{~T}$ then follows a $H^{-0.43}$ dependence in high magnetic fields suggesting different pinning mechanisms in low and high magnetic fields. Similar crossovers were also observed in cuprates and other iron-based superconductors, such as Nd1111, Sm1111 and FeSe11 ${ }^{28-30}$. The weaker field dependence of $U_{0}(H)$ in low fields indicates that single-vortex pinning is dominant. When the magnetic field is increased to a certain amount, the vortex spacing becomes significantly smaller than the penetration depth and there is a crossover to a collective-vortex pinning regime where the activation energy becomes strongly dependent on the field above $7 \mathrm{~T}$, as the collective creep is dominant. The weaker field dependence of $U_{0}$ in the single-vortex pinning causes the larger value of $U_{0}$. However, in the high field region $U_{0}$ tends to decrease faster than with field. Furthermore, the range of $U_{0}$ is much smaller than that reported for single crystal of iron-based superconductors, indicating a lower pinning force for this 1144 polycrystalline sample, as compared with single crystals, which in turn, will affect the values of the critical current densities ${ }^{31-33}$.

\subsection{Critical current properties}

The critical current density, $J_{c}$, is extracted from the isothermal superconducting hysteresis loops, $M-H$, at $2 \mathrm{~K}$ up to $16 \mathrm{~T}$ for all 1144 samples prepared at different $T_{\text {synth }}$. Figure 7(a) shows the $M-H$ loops measured at different temperatures below the critical temperature for the optimum sample. We have not observe the fishtail effect, as found for single crystals $^{3}$. Although the origin of the fishtail effect is not fully explained, however, it is commonly associated with an order-
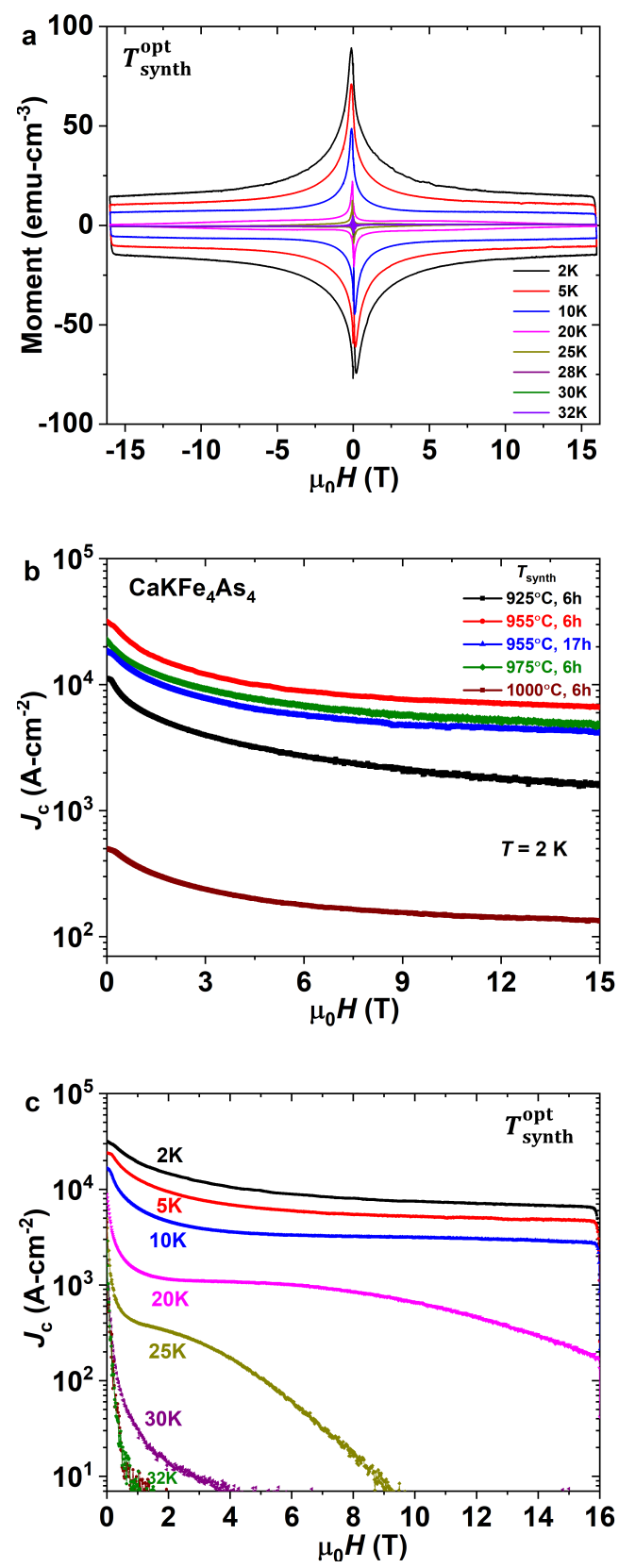

FIG. 7. (a) $M-H$ loops of the polycrystalline sample of $\mathrm{CaKFe}_{4} \mathrm{As}_{4}$ prepared in optimum conditions ( $T_{\mathrm{synth}}^{\mathrm{opt}}$ ) measured inside the superconducting state. (b) The field dependence of the critical current density, $J_{c}$, at $2 \mathrm{~K}$ for different samples heated at $T_{\text {synth. }}$ (c) The variation of the critical current density, $J_{c}$, with respect to the magnetic field for our best 1144 sample (pristine) prepared at $T_{\mathrm{synth}}^{\mathrm{opt}}$.

disorder transition ${ }^{3}$. However, in a polycrystalline sample as compared with single crystals, the distribution of grain sizes as well as the grain boundaries are likely to smear out any features normally seen in single crystal samples, as found in many iron-based superconductors ${ }^{3,34}$. Furthermore, the pinning energy of vortices in the polycrystalline sample is very low and does not exceed the elastic energy in the entire field 

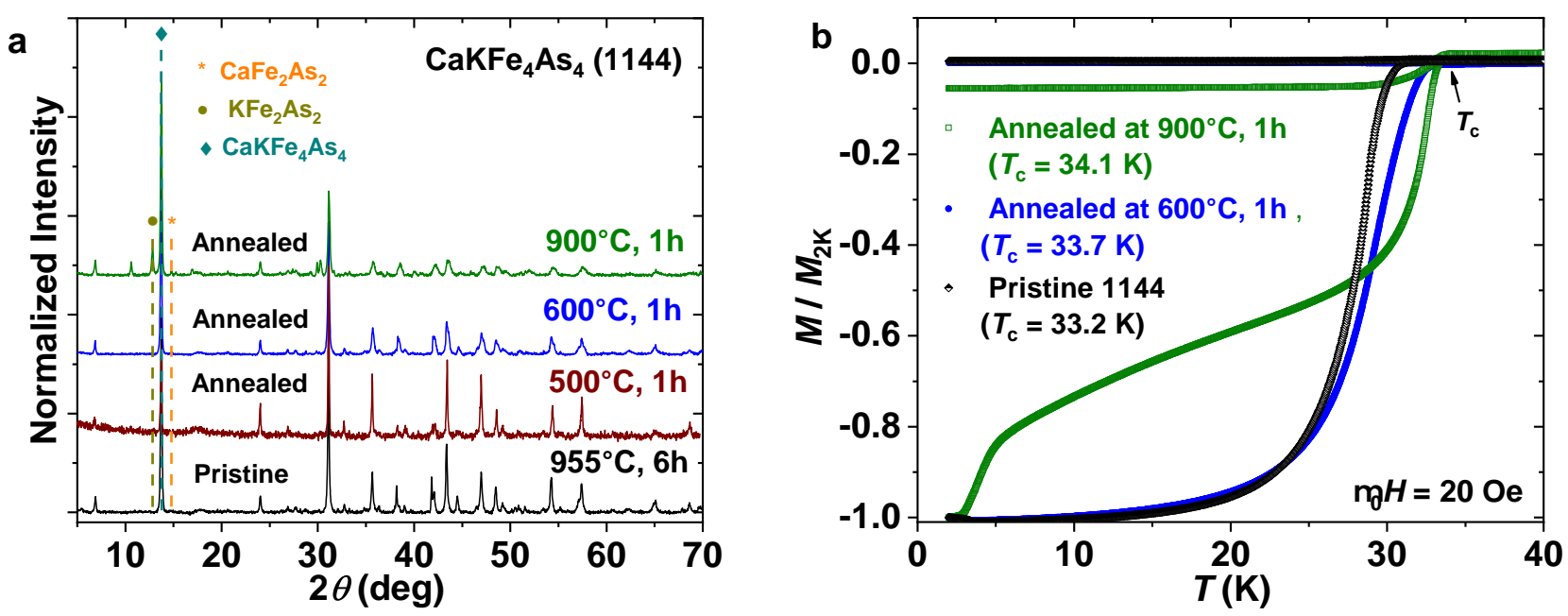

FIG. 8. (a) Powder X-ray diffraction patterns of the optimum sample of $\mathrm{CaKFe}_{4} \mathrm{As}_{4}$ (pristine)) ( $T_{\mathrm{synth}}^{\mathrm{opt}}$ ) annealed at different temperatures. (b) The temperature dependence of the normalized magnetization for the sample post-annealed at $600^{\circ} \mathrm{C}$ and $900^{\circ} \mathrm{C}$.

range in the mixed state, and this may affect also the orderdisorder transition ${ }^{3}$.

An estimation of the critical current density was carried out assuming a homogeneous current flows within the sample using Bean model ${ }^{35}$ with $J_{c}=20 \Delta M / a(a-a / 3 b)$, where $a$ $(\mathrm{cm})$ and $b(\mathrm{~cm})$ are dimensions of the samples with $a<b$ and $\Delta M$ is the variation in magnetization when sweeping the fields down and up for the same magnetic field. The calculated $J_{c}$ for and the field dependence of $J_{c}$ for samples prepared at different synthesis temperature are shown in Figure 7(b). We find higher $J_{c}$ values for the optimum sample compared to the values for the other samples being also similar to that reported value for 1144 wires and tapes ${ }^{14,15}$. The field dependence of $J_{c}$ behaviour for all samples is similar, decreasing rapidly at low fields but becoming weakly field dependent at high fields. As the highest values of $J_{c}$ is obtained for 1144 sample prepared in optimum synthesis conditions $\left(T_{\mathrm{synth}}^{\mathrm{opt}}\right)$, it suggests that this sample has better grain connectivity and quality compared to other samples, as also observed in the resistivity data in Figure 5(a). The field dependence of the calculated $J_{c}$ for this sample at various temperatures is shown in Figure 7(c). It is clear that the $J_{c}$ value at $2 \mathrm{~K}$ is larger than $10^{4} \mathrm{~A} / \mathrm{cm}^{2}$ even at $5 \mathrm{~T}$, and improved compared with other 1144 polycrystalline samples ${ }^{3}$. Such a large value has also been observed in other polycrystalline sample of iron-based superconductors ${ }^{5}$ and it is much larger than that observed in specifically processed C-doped $\mathrm{MgB}_{2}{ }^{36}$.

\subsection{Post-annealing effects}

Our findings discussed above clearly emphasise that the 1144 phase is strongly dependent on preparation conditions and the reaction temperatures. Hence, we have also studied the post-annealing effect of samples prepared in optimum conditions to check the stability of the 1144 phase. The samples are annealed at temperatures ranging from 500 to $900^{\circ} \mathrm{C}$ for a very short time of $\sim 1 \mathrm{~h}$ and the powder diffraction of two samples compared with the pristine sample prepared at $T_{\text {synth }}^{\text {opt }}$ is shown in Figure 8(a). The impurity phases, such as $\mathrm{CaFe}_{2} \mathrm{As}_{2}$ and $\mathrm{KFe}_{2} \mathrm{As}_{2}$, are present in the sample annealed at $900^{\circ} \mathrm{C}$ for $1 \mathrm{~h}$, similar to the initial synthesis process, but the volume fraction of the impurity phases is smaller as the annealing temperature is reduced. For example, the 1144 sample annealed at $600^{\circ} \mathrm{C}$ has very small peaks of impurity phases whereas the sample annealed at $500^{\circ} \mathrm{C}$ has similar patterns to the pristine 1144 as no impurity phases were observed. Small changes in the lattice parameters are observed for the samples annealed at high temperatures, as shown in the Table I. To assess the annealing effect on the superconducting critical temperature $T_{c}$, the temperature dependence of the normalized magnetic moment for post-annealed 1144 samples is compared with the pristine sample, as shown in Figure 8(b). The onset transition temperature for the annealed sample at $900^{\circ} \mathrm{C}$ $\sim$ is $34.1 \mathrm{~K}$ which is slightly higher than that of the pristine sample. Normally, the annealing of the polycrystalline sample at higher temperatures can lead to larger grain size and improvement in intragranular superconducting properties ${ }^{5,37,38}$, in particular the critical current. Furthermore, higher annealing temperature can slightly alter the stoichiometry of the material and lead to the formation of impurity phases ${ }^{2,20,26}$. As observed here, the higher temperature used for annealing is likely to degrade the homogeneity of the material and create additional impurity phases as shown in Figure 8 (a). So, the observed slightly higher $T_{c}$ can be possible due to the small improvement of the grain size $e^{37,38}$ or slightly change in stoichiometry $^{5,26,39}$. However, the shape of the ZFC susceptibility for the post-annealed 1144 at $900^{\circ} \mathrm{C}$ shows two step transitions in Figure 8 (b), much broader in comparison with the pristine sample. This two-step transition is likely to be 

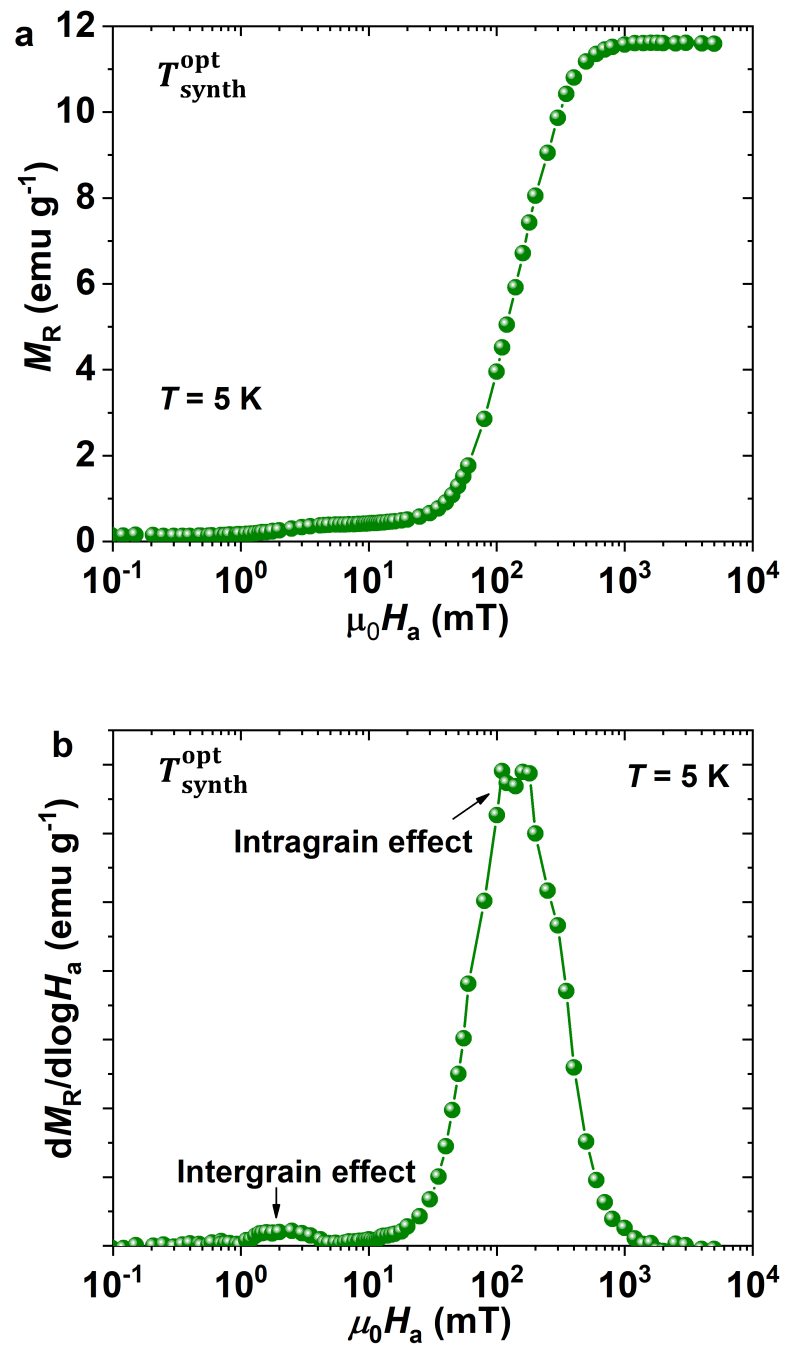

FIG. 9. (a) The magnetic field dependence of remanent magnetization, $M_{R}$ and (b) The derivatives of remanent magnetization with respect to applied magnetic field at $5 \mathrm{~K}$ for an optimum sample of $\mathrm{CaKFe}_{4} \mathrm{As}_{4}$ prepared at $T_{\text {synth }}^{\mathrm{opt}} . M_{R}$ data are normalized by their respective sample masses of $5.7 \mathrm{mg}$.

caused by the 1144 phase and the impurity phase $\mathrm{KFe}_{2} \mathrm{As}_{2}$ which shows a superconducting transition below $\sim 5 \mathrm{~K}^{22}$. Our post-annealing study suggests that the optimum samples start to degrade when annealed above $500^{\circ} \mathrm{C}$ but below this temperature the 1144 phase is stable.

\subsection{Granular nature}

Polycrystalline samples contain a distribution of grains and grain boundaries. These grain boundaries form a network which must be crossed by the supercurrent as it passes through the superconductor. To understand the behaviour of interand intra-granular current density, we investigate the remanent magnetization, $M_{R}$, as a function of increasing applied field for the pure bulk $\mathrm{CaKFe}_{4} \mathrm{As}_{4}$ sample at $5 \mathrm{~K}$, as shown in
Figure 9(a)-(b). When a magnetic field is applied, the flux enters into intergranular regions followed by grain penetration above the lower critical field. The $M_{R}$ for the bulk sample shows two separate steps, similar to that of other iron based superconductors ${ }^{2,40}$. This is better visualized as two separate peaks in the derivative of the remanent magnetization, shown in Figure 9(b), specific to granular superconducting samples $^{34}$. Generally, the first peak at lower fields is related to the breakdown of intergranular shielding characteristics of the individual grains and grain boundaries and strongly depends on the sample size. In this regime, the $M_{R}$ magnitude reflects the local current densities multiplied by the loop over which the supercurrent is flowing ${ }^{17,26,34,40}$. On the other hand, the peak at the higher field is related to the breakdown of intragranular shielding currents of intrinsic nature corresponding to the superconducting state of the individual grains. Figure 9(b) shows that the intergrain peaks are located at $2.3 \mathrm{mT}$ at $5 \mathrm{~K}$, as the magnetic granularity becomes progressively less visible at higher temperatures and there is a better intergrain coupling at lower temperatures. The field dependence of the remanent magnetization can be used to calculate the intergrain current as $J_{c}^{i}=20 \times\left(2 M_{R}\right) /\left(a-a^{2} / 3 b\right)$ using the extended Bean model, as discussed in detail in Refs. 2, 20, and 40. Here, $2 M_{R}$ corresponds to the width of the magnetization hysteresis loop at self field, $H \sim 0$ Oe, and $a$ and $b$ are length and width of bar shaped sample $(a<b)$. We find that the calculated intergrain current, $J_{c}^{i}$ value is $\sim 5.89 \times 10^{2} \mathrm{~A} / \mathrm{cm}^{2}$ at $5 \mathrm{~K}$, much smaller that the values of the total critical current shown in Fig. 7(b).

The intergrain $J_{c}$ is generally affected by the weak-link between grains and strongly decreases with the application of the magnetic field ${ }^{18,19,26}$. Microstructure of the polycrystalline samples is normally responsible for the weak-links between grains and the main obstacle for current blocking are the presence of cracks, impurity phases at the grain boundaries, phase inhomogeneity and the misorientation angle of grain boundaries. ${ }^{17,34}$. We expect that the 1144 are very similar to the 122 phases, which have larger grain boundary angle (up to 10 degree) $)^{17,34}$ compared with cuprates ${ }^{41}$. Furthermore, anisotropy of $\mathrm{CaKFe}_{4} \mathrm{As}_{4}$ is significantly suppressed at low temperatures, as revealed by our recent upper critical field studies ${ }^{42}$. The sample inhomogeneity can arise during cooling from the melt due to the details of the phase diagram, i.e. from thermodynamic and/or kinetic effects, but also can be produced via the intentional introduction of dopant atoms into the host lattice. In this study we are dealing with a stoichiometric superconductor $\mathrm{CaKFe}_{4} \mathrm{As}_{4}$ and its microstructure and compositional analysis along with X-ray diffraction suggest a rather homogeneous and almost clean phase in the polycrystalline samples prepared in optimum conditions. Here the mechanisms responsible for current blocking could be related to the observation of micro and nano pores. The observed pores in 1144 sample may form as a result of thermal stress during the sample preparation due to the quench cooling process. In order to reduce the presence of micro-pores, one needs to optimize the best cooling process for these samples during the synthesis process. As above mentioned, we had tried to cool down the optimal sample to room temperature 
after the reaction with very slow rate but the impurity phases such as $\mathrm{CaFe}_{2} \mathrm{As}_{2}$ and $\mathrm{KFe}_{2} \mathrm{As}_{2}$ were observed. The intergrain $J_{c}$ of $5.89 \times 10^{2} \mathrm{~A} / \mathrm{cm}^{2}$ at $5 \mathrm{~K}$ is very low, despite the chemical homogeneity and the dense and clean phase, due to weak conducting links caused by the presence of micro-pores lying between grains that significantly reduces the current paths. Possible routes to enhance the intergrain $J_{c}$ is via the optimization of heating patterns, metal additions like tin or silver, the densification by high pressure synthesis as well as sintering under ambient pressure or hot isostatic pressing (HIP), and the formation of a $c$-axis grain aligned microstructure ${ }^{17,34}$. Recently, superconducting wires and tapes of 1144 show the transport $J_{c}$ values up to $10^{5} \mathrm{~A}-\mathrm{cm}^{-214,15}$ at the low temperature. These wires were prepared by the hot isostatic pressing (HIP) method which can reduce the formation of pores and lead to well-connected grains at low temperatures, despite the presence of impurity phases in both the polycrystalline powder and the core of the wire. This suggests that to achieve enhanced $J_{c}$ values towards those reported for single crystals $^{3}$, not only the phase purity but also grain connectivity and grain orientation play very important roles. Our optimum 1144 samples show a clean and homogeneous phase but the grain connectivity needs to be significantly improved to enhance the intergrain $J_{c}$. Further studies will be needed to understand and reduce the effect of pores and improvement of grain connectivity in polycrystalline $\mathrm{CaKFe}_{4} \mathrm{As}_{4}$ to increase its critical current density.

\subsection{Synthesis phase diagram}

To summarize the main findings of our study, we present a synthesis phase diagram for this stoichiometric $\mathrm{CaKFe}_{4} \mathrm{As}_{4}$. Figure 10 shows the superconducting transition temperature $T_{c}$ and the critical current density $J_{c}$ as a function of the synthesis temperature, $T_{\text {synth }}$, indicating the formation region of pure 1144 phase. The 1144 sample prepared at $975^{\circ} \mathrm{C}$ has a mixed phase of $\mathrm{CaFe}_{2} \mathrm{As}_{2}$ and $\mathrm{CaKFe}_{4} \mathrm{As}_{4}$ with very small amounts of $\mathrm{KFe}_{2} \mathrm{As}_{2}$. However, as the growth temperature increases, $\mathrm{CaFe}_{2} \mathrm{As}_{2}$ becomes the majority phase, similar to a previous report ${ }^{43}$. These results clearly depict that above $960^{\circ} \mathrm{C}$, the 1144 phase transforms into the $\mathrm{CaFe}_{2} \mathrm{As}_{2}$ phase with very small amount of $\mathrm{KFe}_{2} \mathrm{As}_{2}$ phase and the superconducting properties were suppressed.

Figure 10 indicates that a pure 1144 phase is dominant as the system is prepared around $960^{\circ} \mathrm{C}$. The pure stable phase is obtained around $955^{\circ} \mathrm{C}$ and $T_{c}$ and $J_{c}$ reach the maximum values. Interestingly, the 1144 prepared below $940^{\circ} \mathrm{C}$ becomes again a mixed phase of 122 and 1144 and $\mathrm{KFe}_{2} \mathrm{As}_{2}$ phase is dominant with lowering the temperature (around $900^{\circ} \mathrm{C}$ or below). We observe that $\mathrm{CaFe}_{2} \mathrm{As}_{2}$ appear as a main phase at the higher temperature above $1000^{\circ} \mathrm{C}$ whereas $\mathrm{KFe}_{2} \mathrm{As}_{2}$ become a main phase at temperature below $900^{\circ} \mathrm{C}$. These results are also similar to the previous report ${ }^{22}$ where the optimum synthesis temperature is $900^{\circ} \mathrm{C}$ for $\mathrm{KFe}_{2} \mathrm{As}_{2}$ phase. Yi et al. ${ }^{43}$ suggest that $\mathrm{CaFe}_{2} \mathrm{As}_{2}$ decomposes or melts at very high temperature and this could be possible in our case also

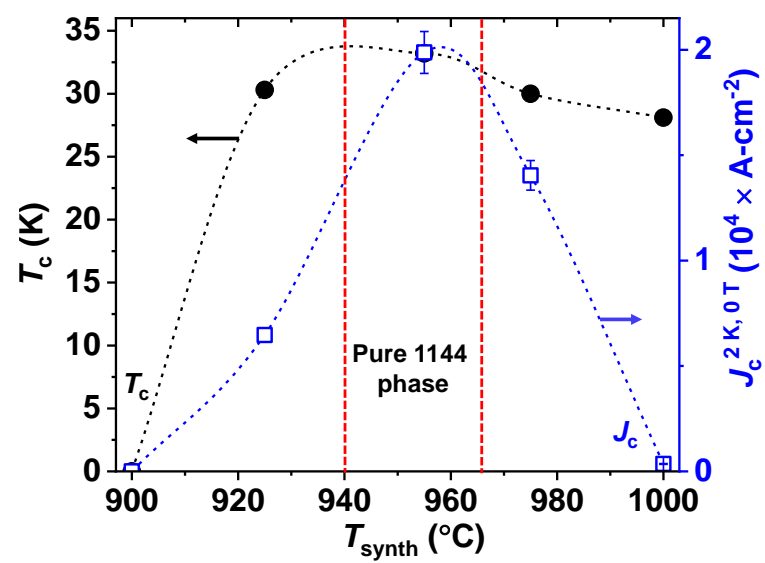

FIG. 10. Synthesis phase diagram for the polycrystalline $\mathrm{CaKFe}_{4} \mathrm{As}_{4}$. The evolution of the critical temperature, $T_{c}$, (left) and the critical current density, $J_{c}$, (right) estimated at $2 \mathrm{~K}$ as a function of the synthesis temperature, $T_{\text {synth }}$. The dash-dotted red lines are guide for the eye to show the optimal synthesis region.

that $\mathrm{CaFe}_{2} \mathrm{As}_{2}$ decomposes into $\mathrm{CaFe}_{4} \mathrm{As}_{3}$ and other phases at higher temperature $\left(1000^{\circ} \mathrm{C}\right)$. The reported phase diagram for the single crystal growth of $\mathrm{CaKFe}_{4} \mathrm{As}_{4}{ }^{13}$ is in good agreement with our findings and it suggests that there is a small temperature window for the growth of the 1144 phase. We also find that the prepared pure 1144 phase further decomposes into the 122 phases during the re-sintering process at above $500^{\circ} \mathrm{C}$ and by increasing the sintering time at the optimum temperature $\left(955^{\circ} \mathrm{C}\right)$.

The present study emphasizes the role of phase purity on the superconducting properties of 1144 . Besides this important element, grain size and grain connectivity are another important factor to the improvement of critical current densities ${ }^{34}$. Other pnictide superconductors, such as the 122 family ${ }^{1,5,6}$, have a larger thermodynamically stable regime of 122 phase formation and annealing processes that improve the grain boundaries and lead to the enhancement of the superconducting properties ${ }^{5,17,44}$. However, 1144 phase has a very narrow synthesis window and they are prone to phase separation into $\mathrm{CaFe}_{2} \mathrm{As}_{2}$ and $\mathrm{KFe}_{2} \mathrm{As}_{2}$. The obtained pristine sample has a clean and homogeneous 1144 phase but due to weak grain boundaries, the obtained the critical current density is very low, as compared with the values found in single crystals ${ }^{3}$ and, in wire and tape ${ }^{14,15}$. However, this synthesis method is suitable for the preparation of high quality and large amounts of powder 1144 that is generally needed for the fabrication of wires and tapes. Further improvement of grains and grain boundaries in 1144 system requires, besides high quality starting material, advanced processing techniques, such as high impact ball milling, high pressure synthesis, ${ }^{4,474}$. 


\section{CONCLUSIONS}

In summary, $\mathrm{CaKFe}_{4} \mathrm{As}_{4}$ polycrystalline samples have been synthesized in a wide synthesis temperature range (900$1100^{\circ} \mathrm{C}$ ) by the solid state reaction method and characterized by magnetic and transport measurements. Our results indicate that 1144 phase is sensitive with respect to the synthesis temperature and time. Various characterization methods indicate that the optimum superconducting properties with the largest critical current densities, low resistivity values, sharp superconducting transition, large residual resistivity ratio are obtained when synthesizing at a temperature of $955^{\circ} \mathrm{C}$ for $6 \mathrm{~h}\left(T_{\mathrm{synth}}^{\mathrm{opt}}\right)$. This optimum synthesis conditions lead to the formation of a phase pure polycrystalline samples and small temperature differences of $20-25^{\circ} \mathrm{C}$ generates impurity phases in the form of 122 phases. A microstructural investigation and critical current densities values of the optimum sample revealed that superconducting grains have a disc shape and better grain connectivity compared to other synthesis conditions. The remanent magnetization study demonstrates the coupled effect of inter- and intragranular behaviour due to the origin of weak Josephson coupling between grains and confirms the granular nature of this 1144 compound similar to other iron-based superconductors. The synthesis phase diagram is in good agreement with that reported for single crystals of 1144. Our study provides the details of a promising synthesis route to develop high quality polycrystalline samples in order to help the development and the fabrication of the tapes and wires of this promising material.

Acknowledgements. The research was funded by the Oxford Centre for Applied Superconductivity (CFAS) at Oxford University. We also acknowledge EPSRC grants Diamond Light Source allocation and financial support of the John Fell Fund of the Oxford University. AIC acknowledges an EPSRC Career Acceleration Fellowship (EP/I004475/1).
* corresponding author: shiv.singh@physics.ox.ac.uk

$\dagger$ corresponding author: amalia.coldea@physics.ox.ac.uk

${ }^{1}$ H. Hosono, K. Tanabe, E. Takayama-Muromachi, H. Kageyama, S. Yamanaka, H. Kumakura, M. Nohara, H. Hiramatsu, and S. Fujitsu, "Exploration of new superconductors and functional materials, and fabrication of superconducting tapes and wires of iron pnictides," Sci. Technol. Adv. Mater. 16, 033503 (2015).

2 S. J. Singh, J. Shimoyama, A. Yamamoto, H. Ogino, and K. Kishio, "Transition Temperature and Upper Critical Field in $\mathrm{SmFeAsO} \mathrm{O}_{1-x} \mathrm{~F}_{x}$ Synthesized at Low Heating Temperatures," IEEE Trans. Appl. Supercond. 23, 8 (2013).

${ }^{3}$ Shiv J. Singh, Matthew Bristow, William R. Meier, Patrick Taylor, Stephen J. Blundell, Paul C. Canfield, and Amalia I. Coldea, "Ultrahigh critical current densities, the vortex phase diagram, and the effect of granularity of the stoichiometric high- $T_{c}$ superconductor $\mathrm{CaKFe}_{4} \mathrm{As}_{4}$," Phys. Rev. Materials 2, 074802 (2018).

${ }^{4}$ Hideo Hosono, Akiyasu Yamamoto, Hidenori Hiramatsu, and Yanwei Ma, "Recent advances in iron-based superconductors toward applications," Materials Today 21, 278 (2018).

5 Chao Yao and Yanwei Ma, "Recent breakthrough development in iron-based superconducting wires for practical applications," Superconductor Science and Technology 32, 023002 (2019).

${ }^{6}$ M. Rotter, M. Tegel, and D. Johrendt, "Superconductivity at 38 $\mathrm{K}$ in the iron arsenide $\mathrm{Ba}_{1-x} \mathrm{~K}_{x} \mathrm{Fe}_{2} \mathrm{As}_{2}$," Phys. Rev. Lett. 101, 107006 (2008).

7 A. Iyo, K. Kawashima, T. Kinjo, T. Nishio, S. Ishida, H. Fujihisa, Y. Gotoh, K. Kihou, H. Eisaki, and Y. Yoshida, "New-StructureType Fe-Based Superconductors: $\mathrm{CaKFe}_{4} \mathrm{As}_{4}(\mathrm{~A}=\mathrm{K}, \mathrm{Rb}, \mathrm{Cs})$ and $\mathrm{SrAFe}_{4} \mathrm{As}_{4}(\mathrm{~A}=\mathrm{Rb}, \mathrm{Cs})$," J. Am. Chem. Soc. 138, 3410 (2016).

8 W. R. Meier, T. Kong, U. S. Kaluarachchi, V. Taufour, N. H. Jo, G. Drachuck, A. E. Böhmer, S. M. Saunders, A. Sapkota, A. Kreyssig, M. A. Tanatar, R. Prozorov, A. I. Goldman, F. F. Balakirev, A. Gurevich, S. L. Bud'ko, and P. C. Canfield, "Anisotropic thermodynamic and transport properties of single crystalline CaKFe ${ }_{4} \mathrm{As}_{4}$," Phys. Rev. B 94, 064501 (2016).

9 Kyuil Cho, A. Fente, S. Teknowijoyo, M. A. Tanatar, K. R. Joshi, N. M. Nusran, T. Kong, W. R. Meier, U. Kaluarachchi, I. Guillamón, H. Suderow, S. L. Bud'ko, P. C. Canfield, and R. Prozorov, "Nodeless multiband superconductivity in stoichiometric single-crystalline $\mathrm{CaKFe}_{4} \mathrm{As}_{4}$," Phys. Rev. B 95, 100502 (2017).

${ }^{10}$ H. Q. Yuan, J. Singleton, F. F. Balakirev, S. A. Baily, G. F. Chen, J. L. Luo, and N. L. Wang, "Nearly isotropic superconductivity in (Ba,K)Fe ${ }_{2} \mathrm{As}_{2}$," Nature 457, 565 (2009), 0807.3137.

11 N. Ni, S. L. Bud'ko, A. Kreyssig, S. Nandi, G. E. Rustan, A. I. Goldman, S. Gupta, J. D. Corbett, A. Kracher, and P. C. Canfield, "Anisotropic thermodynamic and transport properties of singlecrystalline BaK122,” Phys. Rev. B 78, 014507 (2008).

12 R. Lortz, N. Musolino, Y. Wang, A. Junod, and N. Toyota, "Origin of the magnetization peak effect in the $\mathrm{Nb}_{3} \mathrm{Sn}$ superconductor," Phys. Rev. B 75, 094503 (2007).

13 W. R. Meier, T. Kong, S. L. Bud'ko, and P. C. Canfield, "Optimization of the crystal growth of the superconductor $\mathrm{CaKFe}_{4} \mathrm{As}_{4}$ from solution in the FeAs-CaFe $\mathrm{As}_{2}-\mathrm{KFe}_{2} \mathrm{As}_{2}$ system," Phys. Rev. Materials 1, 013401 (2017).

14 Zhe Cheng, Chiheng Dong, He Huang, Shifa Liu, Yanchang Zhu, Dongliang Wang, Vitalii Vlasko-Vlasov, Ulrich Welp, WaiKwong Kwok, and Yanwei Ma, "Chemical stability and superconductivity in ag-sheathed $\mathrm{CaKFe}_{4} \mathrm{As}_{4}$ superconducting tapes," Superconductor Science and Technology 32, 015008 (2018).

15 Sunseng Pyon, Daisuke Miyawaki, Ivan Veshchunov, Tsuyoshi Tamegai, Katsutoshi Takano, Hideki Kajitani, Norikiyo Koizumi, and Satoshi Awaji, "Fabrication and characterization of $\mathrm{CaKFe}_{4} \mathrm{As}_{4}$ round wires sintered at high pressure," Applied Physics Express 11, 123101 (2018).

16 T. Roisnel and J. Rodriguez-Carvajal, "Winplotr: A windows tool for powder diffraction pattern analysis," Mater. Sci. Forum 118, 378-381 (2001).

17 M. Putti, I. Pallecchi, E. Bellingeri, M. Tropeano, C. Ferdeghini, A. P. C. Tarantini, A. Yamamoto, J. Jiang, J. Jaroszynski, F. Kametani, D. Abraimov, A. Polyanskii, J. D. Weiss, E. E. Hellstrom, A. Gurevich, D. C. Larbalestier, R. Jin, B. C. Sales, A. S. Sefat, M. A. McGuire, D. Mandrus, P. Cheng, Y. Jia, H. H. Wen, S. Lee, and C. B. Eom, "New Fe-based superconductors: properties relevant for applications," Supercond. Sci. Technol. 23, 034003 (2009).

18 F. Kametani, P. Li, D. Abraimov, A. A. Polyanskii, A. Yamamoto, J. Jiang, E. E. Hellstrom, A. Gurevich, D. C. Larbalestier, Z. A. Ren, J. Yang, X. L. Dong, W. Lu, and Z. X. Zhao, "Intergrain current flow in a randomly oriented polycrystalline $\mathrm{SmFeAsO}_{0.85}$ 
oxypnictide," Applied Physics Letters 95, 1-4 (2009).

19 Fumitake Kametani, A A Polyanskii, A Yamamoto, J Jiang, E E Hellstrom, A Gurevich, D C Larbalestier, Z A Ren, J Yang, X L Dong, W Lu, and $\mathrm{Z} \mathrm{X} \mathrm{Zhao,} \mathrm{"Combined} \mathrm{microstructural} \mathrm{and}$ magneto-optical study of current flow in polycrystalline forms of Nd and Sm Fe-oxypnictides," Supercond. Sci. Technol. 22, 015010 (2008).

${ }^{20}$ Shiv J. Singh, Jun ichi Shimoyama, Akiyasu Yamamoto, Hiraku Ogino, and Kohji Kishio, "Significant enhancement of the intergrain coupling in lightly F-doped $\mathrm{SmFeAsO}$ superconductors," Supercond. Sci. Technol. 26, 065006 (2013).

${ }^{21}$ S. R. Saha, N. P. Butch, T. Drye, J. Magill, S. Ziemak, K. Kirshenbaum, P. Y. Zavalij, J. W. Lynn, and J. Paglione, "Structural collapse and superconductivity in rare-earth-doped $\mathrm{CaFe}_{2} \mathrm{As}_{2}$," Phys. Rev. B 85, 024525 (2012).

${ }^{22}$ Kalyan Sasmal, Bing Lv, Bernd Lorenz, Arnold M. Guloy, Feng Chen, Yu-Yi Xue, and Ching-Wu Chu, "Superconducting febased compounds $\left(\mathrm{A}_{1-x} \mathrm{Sr}_{x}\right) \mathrm{Fe}_{2} \mathrm{As}_{2}$ with $\mathrm{A}=\mathrm{K}$ and $\mathrm{Cs}$ with transition temperatures up to $37 \mathrm{~K}$," Phys. Rev. Lett. 101, 107007 (2008).

23 D. X. Chen, E. Pardo, and A. Sanchez, "Demagnetizing factors of rectangular prisms and ellipsoids," IEEE Trans. Magnet. 38, 1742 (2002).

${ }^{24}$ M. S. Torikachvili, S. L. Bud'ko, Ni Ni, and P. C. Canfield, "Pressure induced superconductivity in $\mathrm{CaFe}_{2} \mathrm{As}_{2}$," Phys. Rev. Lett. 101, 057006 (2008).

25 N. R. Werthamer, E. Helfand, and P. C. Hohenberg, "Temperature and purity dependence of the superconducting critical field, $H_{c 2}$. III. electron spin and spin-orbit effects," Phys. Rev. 147, 295-302 (1966).

${ }^{26}$ Shiv J. Singh, Robert Beck, Sabine Wurmehl, Christian Hess, and Bernd Büchner, "Granular behavior observed in the polycrystalline superconducting LiFeAs," Supercond. Sci. Technol. 28, 025006 (2015).

27 T. Matsushita and B. Ni, "Broad resistive transition under magnetic field in high-temperature superconductors," Physica C 166, 423 (1990).

${ }^{28}$ H.-S. Lee, M. Bartkowiak, J. S. Kim, and H.-J. Lee, "Magneticfield-induced crossover of vortex-line coupling in $\mathrm{SmFeAsO}_{0.85}$ single crystal," Phys. Rev. B 82, 104523 (2010).

${ }^{29}$ H. Lei, R. Hu, E. S. Choi, and C. Petrovic, "Thermally activated energy and flux-flow hall effect of Fe(Te,Se), Phys. Rev. B 82, 134525 (2010)

30 J. Jaroszynski, F. Hunte, L. Balicas, Youn-jung Jo, I. Raičević, A. Gurevich, D. C. Larbalestier, F. F. Balakirev, L. Fang, P. Cheng, Y. Jia, and H. H. Wen, "Upper critical fields and thermallyactivated transport of $\mathrm{NdFeAsO}_{0.7} \mathrm{~F}_{0.3}$ single crystal," Phys. Rev.
B 78, 174523 (2008).

31 X.-L. Wang, S. R. Ghorbani, S.-I. Lee, S. X. Dou, C. T. Lin, T. H. Johansen, K.-H. Müller, Z. X. Cheng, G. Peleckis, M. Shabazi, A. J. Qviller, V. V. Yurchenko, G. L. Sun, and D. L. Sun, "Very strong intrinsic flux pinning and vortex avalanches in (Ba, K) $\mathrm{Fe}_{2} \mathrm{As}_{2}$ superconducting single crystals," Phys. Rev. B 82, 024525 (2010).

${ }^{32}$ H.-S. Lee, M. Bartkowiak, J. S. Kim, and Hu-J. Lee, "Magneticfield-induced crossover of vortex-line coupling in $\mathrm{SmFeAsO}_{0.85}$ single crystal," Phys. Rev. B 82, 104523 (2010).

33 V. A. Vlasenko, K. S. Pervakov, and S. U. Gavrilkin, "Vortex dynamic, pinning and irreversibility field investigation in $\mathrm{EuRbFe}_{4} \mathrm{As}_{4}$ superconductor," (2019), arXiv:1906.04597.

34 J H Durrell, C-B Eom, A Gurevich, E E Hellstrom, C Tarantini, A Yamamoto, and D C Larbalestier, "The behavior of grain boundaries in the Fe-based superconductors," Reports on Progress in Physics 74, 124511 (2011).

35 P. C. Bean, "Magnetization of High-Field Superconductors," Rev. Mod. Phys. 36, 31 (1985).

${ }^{36} \mathrm{C}$. Buzea and T. Yamashita, "Review of the superconducting properties of $\mathrm{MgB}_{2}$," Supercond. Sci. Technol. 14, R115 (2001).

${ }^{37}$ S. Bose, P. Raychaudhuri, R. Banerjee, P. Vasa, and P. Ayyub, "Mechanism of the size dependence of the superconducting transition of nanostructured Nb,” Phys. Rev. Lett. 95, 147003 (2005).

38 Y. Jin, X. Song, and D. Zhang, "Grain-size dependence of superconductivity in dc sputtered nb films," Science in China Series G: Physics, Mechanics and Astronomy 52, 1289-1292 (2009).

39 I. Pallecchi, M. Eisterer, A. Malagoli, and M. Putti, "Application potential of fe-based superconductors," Superconductor Science and Technology 28, 114005 (2015).

40 A. Yamamoto, J. Jaroszynski, C. Tarantini, L. Balicas, J. Jiang, A. Gurevich, D. C. Larbalestier, R. Jin, A. S. Sefat, M. A. McGuire, B. C. Sales, D. K. Christen, and D. Mandrus, "Small anisotropy, weak thermal fluctuations, and high field superconductivity in Co-doped iron pnictide $\mathrm{Ba}\left(\mathrm{Fe}_{1-x} \mathrm{Co}_{x}\right)_{2} \mathrm{As}_{2}$," Appl. Phys. Lett. 94, 98 (2009).

${ }^{41}$ H. Hilgenkamp and J. Mannhart, "Grain boundaries in high- $T_{c}$ superconductors," Rev. Mod. Phys. 74, 485-549 (2002).

${ }^{42}$ M. Bristow et al., In preparation (2019).

43 Tanghong Yi, Adam P. Dioguardi, Peter Klavins, Nicholas J. Curro, Liang L. Zhao, E. Morosan, and Susan M. Kauzlarich, "Synthesis and thermal stability studies of $\mathrm{CaFe}_{4} \mathrm{As}_{3}$," European Journal of Inorganic Chemistry , 3920-3925 (2011).

44 J. D. Weiss, J. Jiang, A. A. Polyanskii, and E. E. Hellstrom, "Mechanochemical synthesis of pnictide compounds and superconducting $\mathrm{Ba}_{0.6} \mathrm{~K}_{0.4} \mathrm{Fe}_{2} \mathrm{As}_{2}$ bulks with high critical current density," Supercond. Sci. Technol. 26, 074003 (2013). 